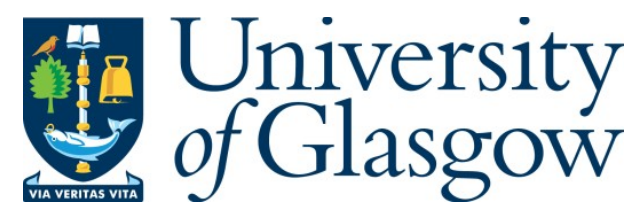

Moia, D. et al. (2019) Ionic-to-electronic current amplification in hybrid perovskite solar cells: ionically gated transistor-interface circuit model explains hysteresis and impedance of mixed conducting devices. Energy and Environmental Science, 12(4), pp. 1296-1308.

There may be differences between this version and the published version. You are advised to consult the publisher's version if you wish to cite from it; https://doi.org/10.1039/C8EE02362J

This journal is (c) The Royal Society of Chemistry 2019.This is the author's version of the work. It is posted here for your personal use. Not for redistribution.

http://eprints.gla.ac.uk/223139/

Enlighten - Research publications by members of the University of Glasgow http://eprints.gla.ac.uk 


\title{
Ionic-to-electronic current amplification in hybrid perovskite solar cells: ionically gated transistor-interface circuit model explains hysteresis and impedance of mixed conducting devices
}

Davide Moia ${ }^{1+*}$, Ilario Gelmetti ${ }^{2,3{ }^{*}}$, Phil Calado ${ }^{1}$, William Fisher ${ }^{1}$, Michael Stringer ${ }^{4}$, Onkar Game ${ }^{4}$, Yinghong Hu5, Pablo Docampo5,6, David Lidzey, Emilio Palomares²,7, Jenny Nelson¹, Piers R. F. Barnes $^{1 *}$

1Department of Physics, Imperial College London, London SW7 2AZ, UK

2Institute of Chemical Research of Catalonia (ICIQ), Barcelona Institute of Science and Technology (BIST), Avda. Països Catalans 16, 43007 Tarragona, Spain

${ }^{3}$ Departament d'Enginyeria Electrònica, Elèctrica i Automàtica, Universitat Rovira i Virgili, Avda. Països Catalans 26, 43007 Tarragona, Spain

${ }^{4}$ Department of Physics and Astronomy, University of Sheffield, Sheffield S3 7RH, UK

5Department of Chemistry and Center for NanoScience (CeNS), LMU München, Butenandtstrasse 5-13, 81377 München, Germany

6Physics Department, School of Electrical and Electronic Engineering, Newcastle University, Newcastle upon Tyne NE1 7RU, UK

7ICREA, Passeig Lluís Companys, 23, Barcelona, Spain

* d.moia@fkf.mpg.de

* igelmetti@iciq.es

† These authors contributed equally to this study

\begin{abstract}
Mobile ions in hybrid perovskite semiconductors introduce a new degree of freedom to electronic devices suggesting applications beyond photovoltaics. An intuitive device model describing the interplay between ionic and electronic charge transfer is needed to unlock the full potential of the technology. We describe the perovskite-contact interfaces as transistors which couple ionic charge redistribution to energetic barriers controlling electronic injection and recombination. This reveals an amplification factor between the out of phase electronic current and the ionic current. Our findings suggest a strategy to design thin film electronic components with large, tuneable, capacitor-like and inductor-like characteristics. The resulting simple equivalent circuit model, which we verified with time-dependent drift-diffusion simulations of measured impedance spectra, allows a general description and interpretation of perovskite solar cell behaviour.
\end{abstract}

\section{Broader context}

Highly efficient solar cells made using hybrid perovskite semiconductors may prove commercially viable. The success of these cheap materials is in part due to their ability to 
tolerate high concentrations of crystal defects associated with processing at low temperature while retaining excellent electronic properties. The presence of these electrically charged defects, some of which are mobile, has an interesting side-effect: the solar cell performance will vary with time following a change in the operating conditions (often referred to as hysteresis). This significantly complicates the measurement and analysis of these materials. Hysteresis means that the diode equivalent circuit model, which is successfully used as the simplest description of virtually all other photovoltaic technologies, is not applicable to most hybrid perovskite devices. We show that the interfaces of solar cells and related devices containing inert mobile ions can be very well described if the diode model is replaced by a transistor model. In this description, the 'gate' of the transistor is controlled by the accumulation of mobile charged defects. Consequently, if the time dependent variation of the ionic charge can be understood then the electrical properties of the device can be predicted. This powerful model provides a framework to allow new material/interfaces to be screened for solar cells and other devices laden with inert mobile defects, it also provides a means to unlock the potential of impedance spectroscopy for characterisation, and a method to determine ionic conductivities in hybrid perovskites.

\section{Table of contents graphic}

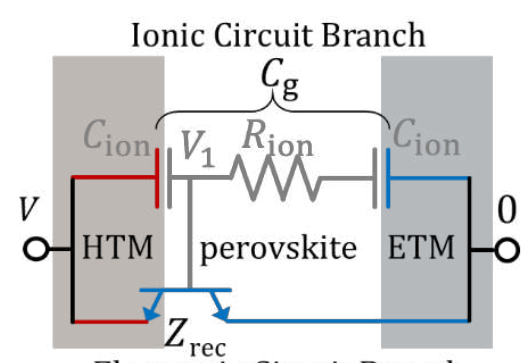

Electronic Circuit Branch

The time and frequency dependent behaviour of hybrid perovskite solar cells is described by an interfacial-transistor circuit model which couples electronic charge transfer to mobile ions. 


\section{Main Text}

The exponential increase in current with the voltage applied across a semiconductor junction arises from the asymmetric change in the energy barrier to charge transfer in each direction across the junction (Fig. 1 ). This realisation was a pivotal step in human history. It underpinned the success of the diode and led to the development of transistors and optoelectronic devices such as light emitting diodes and solar cells. Representing solar cells as diodes in equivalent circuit models neatly encapsulates their behaviour ${ }^{1}$ and has helped facilitate the worldwide deployment of photovoltaics. However, solar cells based on the rapidly developing technology of hybrid perovskite semiconductors ${ }^{2,3}$ do not generally display pure diode-like characteristics. Identifying an accurate equivalent circuit model describing their behaviour is a priority, both to unravel their unique history-dependent properties, and to enable development and application of new electronic devices utilising these properties. Mobile ionic defects in the perovskite semiconductor phase are thought to underlie the hysteresis often seen in current-voltage sweeps and step-measurements characteristics ${ }^{4-7}$ but a physically meaningful equivalent circuit explaining the very large capacitive $\left(>10^{-3} \mathrm{~F} \mathrm{~cm}^{-2}\right)$ and inductive $\left(>1 \mathrm{H} \mathrm{cm}^{-2}\right.$ ) behaviour reported in perovskite devices is lacking, ${ }^{3-12}$. Ferroelectric effects, a photoinduced giant dielectric constant ${ }^{13}$, and accumulation of ionic charge $\mathrm{e}^{7,14}$ have all been discounted as explanations ${ }^{15-17}$. Bisquert et al. have proposed that giant capacitances and inductances ${ }^{17-19}$ could arise from phase-shifted accumulation or release of electronic charge from within a degenerate layer induced by fluctuations in the surface polarisation due to ionic charge. However, interfacial degeneracy is unlikely to exist under normal operating conditions..$^{20}$ More promisingly, Pockett et al. have highlighted the link between rate of recombination and varying ion distribution as an explanation for the low frequency behaviour of perovskite impedance spectra ${ }^{10}$. Previous attempts to model the interaction between electronic and ionic charge have used capacitive elements which cannot describe the influence of one species on the electrostatic potential barriers that control fluxes of the other species. This intrinsically limits the applicability of equivalent circuit models of mixed conductors such as perovskites. 
a Diode

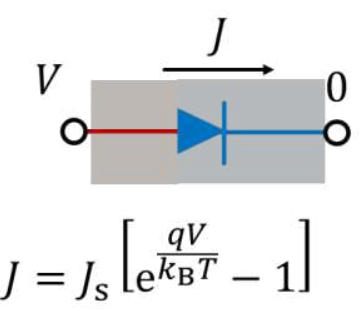

b Bipolar Transistor

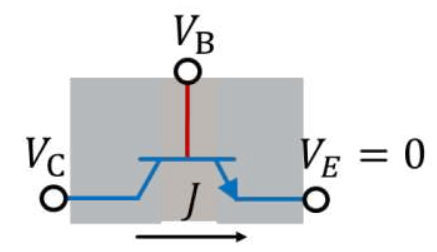

$J=J_{\mathrm{S}}\left[\mathrm{e}^{\frac{q\left(V_{\mathrm{B}}-V_{\mathrm{E}}\right)}{k_{\mathrm{B}} T}}-\mathrm{e}^{\frac{q\left(V_{\mathrm{B}}-V_{\mathrm{C}}\right)}{k_{\mathrm{B}} T}}\right]$

c Mixed Conductor Diode

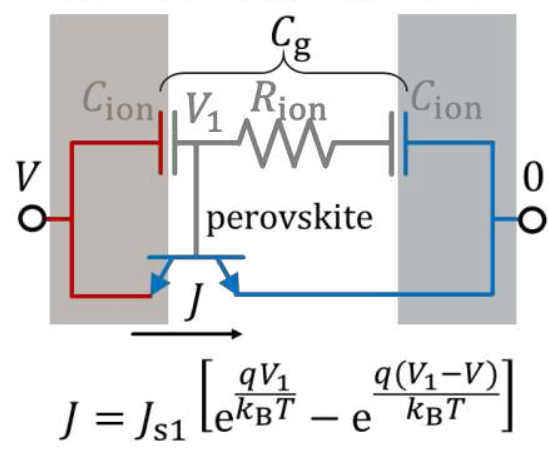
— hole conduction dominant electron conduction dominant ionic conduction
$\mathrm{p}$ - type material
$\mathrm{n}$ - type material
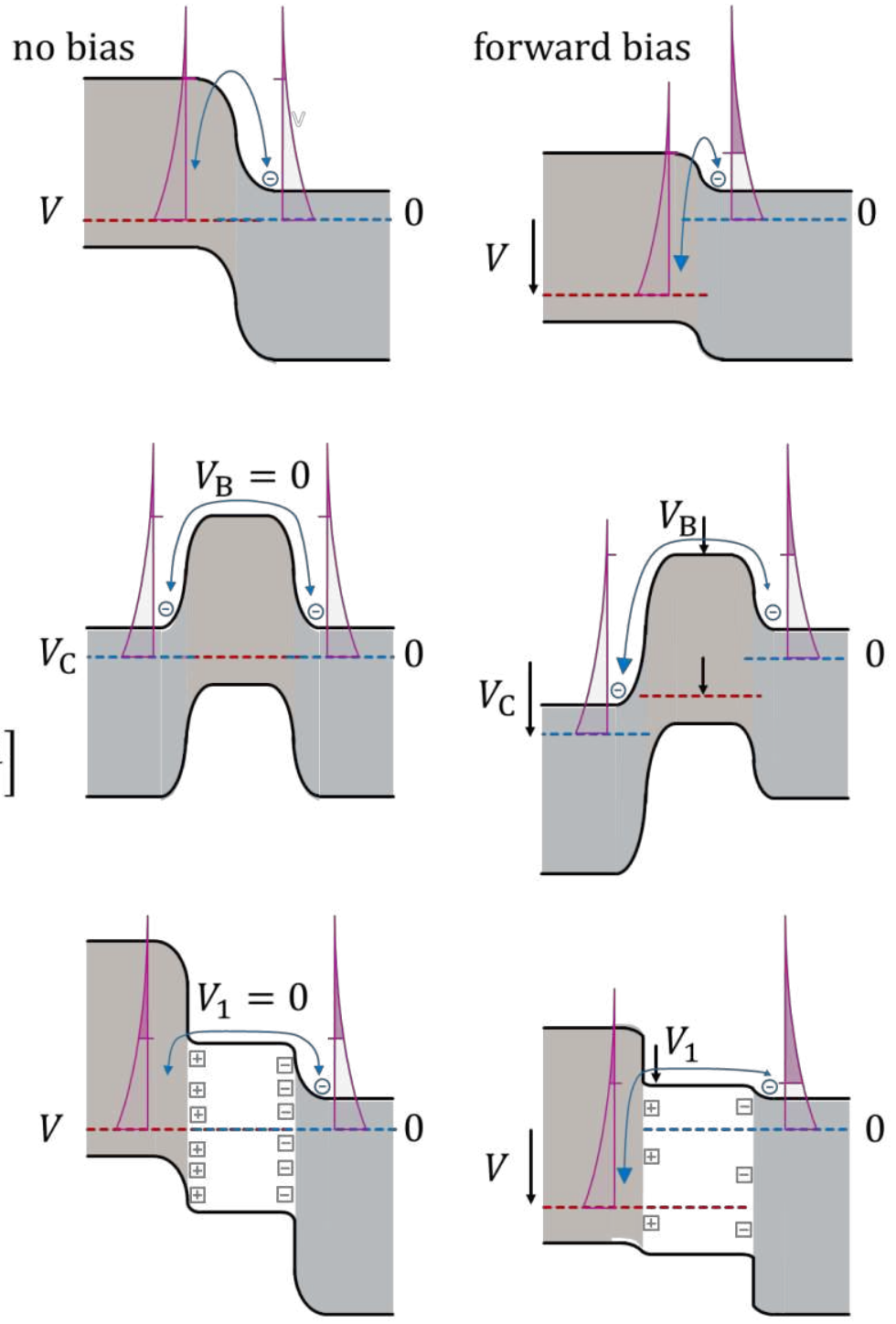

---- hole (quasi) Fermi level

---- electron (quasi) Fermi level

- conduction/valence band

$\boxplus$ positive ionic charge

$\boxminus$ negative ionic charge fraction of electrons $(\Theta)$ in with sufficient energy to overcome device energy barrier Boltzmann distribution

Fig. 1 Device circuit models and schematic energy level diagrams of a diode, bipolar transistor, and perovskite solar cell in their unbiased and forward biased steady

states. In the energy level diagrams, a positive voltage difference is in the downward direction. All voltages are referenced to the Fermi level of the n-type material on the right-hand side (in contact with the cathode) defined to be zero. The difference in magnitude of the flux of electrons across the energy barrier in each direction is indicated by relative size of the arrow heads on the curved blue lines. (a) A p-n junction diode. A forward bias voltage applied across the diode reduces the barrier to electron transfer from the n-type region by $V$, exponentially increasing the flux in this direction, while the flux from the p-type region is unchanged $U_{s}$ ) resulting in a total current density $J$. (b) A 
bipolar n-p-n transistor where the electrical potentials on collector, base and emitter terminals are $V_{\mathrm{C}}, V_{\mathrm{B}}$ and $V_{\mathrm{E}}$ respectively, we define $V_{\mathrm{E}}=0$ and the base to collector current gain to be infinite. In the unbiased state $V_{\mathrm{C}}=V_{\mathrm{B}}=V_{\mathrm{E}}=0$. The barrier height of the p-type region can be modulated by varying $V_{\mathrm{B}}$ which exponentially changes the flux of electrons overcoming the barrier from each side, resulting in a total current between the collector and emitter of $J$. The recombination of electrons with holes in the base is neglected. (c) A perovskite solar cell forming a mixed ionic-electronic conducting diode. Changes from the dark equilibrium distribution of mobile ionic charge (which occurs with time constant $R_{\mathrm{ion}} C_{\mathrm{ion}} / 2$ ) result in a change in electrostatic potential, $V_{1}$, relative to dark equilibrium. This gates electronic charge transfer across the perovskite HTM interface in a manner analogous to the base of a bipolar transistor (c.f. b). The overall device has only two external terminals, $V_{1}$ is voltage on the base of the transistor element in the circuit model, $J_{\mathrm{s} 1}$ is the saturation current density of interface at dark equilibrium.

Here we show that the interfaces in perovskite solar cells behave like bipolar transistors ${ }^{21}$ Fig. 1 1) in which the electronic energy barriers to injection and recombination are modulated by the accumulation/depletion of ionic charge at the interfaces Fig. 1 ${ }^{22}$. Using drift-diffusion simulations of impedance measurements which include mobile ions, we find that: (i) an oscillating voltage applied to the solar cell naturally introduces an out of phase, capacitive ionic current; (ii) the associated changes in electrostatic potential from ion redistribution across the perovskite modulate the rates of electronic recombination and injection across the interfaces. The resulting out of phase electronic current is related to the ionic current through a transcarrier amplification factor with either a positive or negative sign (depending on the recombination or injection mechanisim dominating the device's impedance) and causes capacitor-like or inductor-like behaviour without accumulation of electronic charge at the interfaces. Modelling this amplification effect using ionically gated transistor elements incorporated in a simple equivalent circuit Fig. 14) allows us to efficiently explain and physically interpret the many peculiar features observed in the small and large perturbation transient behaviour of perovskite devices (including impedance and current-voltage sweeps). In this context ionic gating is the control of the electronic charge transfer rate across an interface in response to changes in electrostatic potential from mobile ionic charge in the active layer.

The ionically gated interface-transistor model for mixed conductor devices has similar explanatory power to the diode model applied to standard semiconductor devices. It incorporates the key physics of the device to provide a coherent general description of both the time, and frequency dependent behaviour of perovskite solar cells. The resulting insights open the possibility of engineering a new class of mixed conducting electronic devices whose behaviour is controlled by the properties of mobile ions in the active layer. It also reduces the need for far more complex drift-diffusion models and enables key performance parameters of 
interfaces to be meaningfully assessed using techniques such as electrochemical impedance spectroscopy.

\section{Measured and simulated impedance spectra characteristics}

To demonstrate the application of the interface-transistor model and the ionic-to-electronic current amplification behaviour at device interfaces we measured impedance spectra of perovskite solar cells. Impedance spectroscopy involves the application of a voltage, $V$, across the external terminals of the device, which includes small periodic voltage perturbation, $v$, superimposed on a background voltage, $\bar{V}$, and measurement of the amplitude and phase shift of the induced oscillating current, $j$, superimposed on a background current $\bar{J}$. The complex impedance $\left(Z=Z^{\prime}+i Z^{\prime \prime}\right)$ is given by $Z=|v / j| \exp (\mathrm{i} \theta)$ where $\theta$ is the phase shift of $v$ relative to $j$. This is evaluated at different angular frequencies $(\omega)$ resulting in a spectrum $Z(\omega)$.

Fig. 2 and b show impedance data collected from a stable perovskite solar cell equilibrated at open circuit for different light intensities (see complete spectra in Fig. S1, ESI and the effects of stabilisation time which reduces loop artefacts in Fig. S2a-d, ESI). The measurements indicate that, at low frequencies, there is a significant out of phase component in the induced current $\left(j^{\prime \prime}\right)$ which results in a large apparent device capacitance, as defined by $\omega^{-1} \operatorname{Im}\left(Z^{-1}\right)$. This increases linearly with light intensity and thus exponentially with the bias voltage (Fig. 2p), consistent with previous observations $8,11,18,23$. Similar behaviour was also seen at short circuit, or with different applied biases in the dark (Fig. S2d, e, h, i, ESI) ruling out a significant contribution from photoinduced changes in ionic conductivity 24,25 (Fig. S3, ESI).
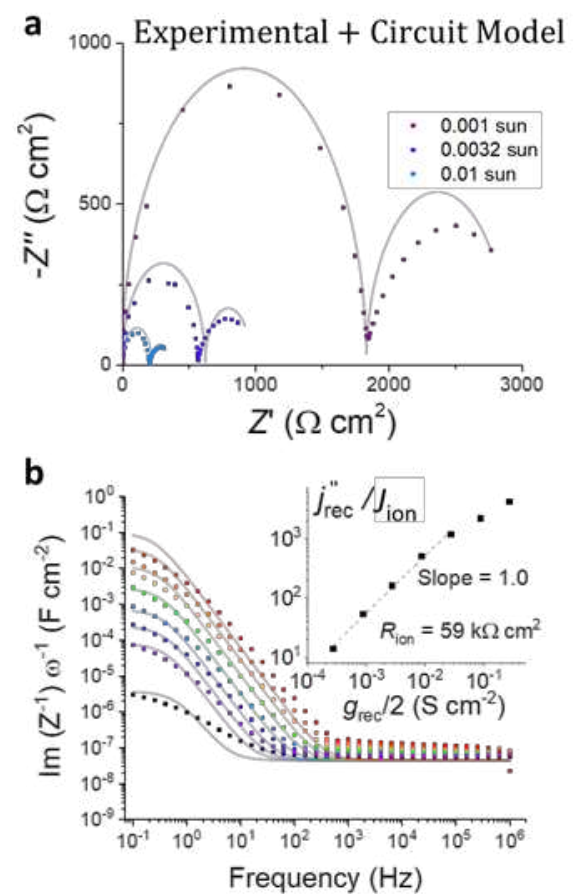

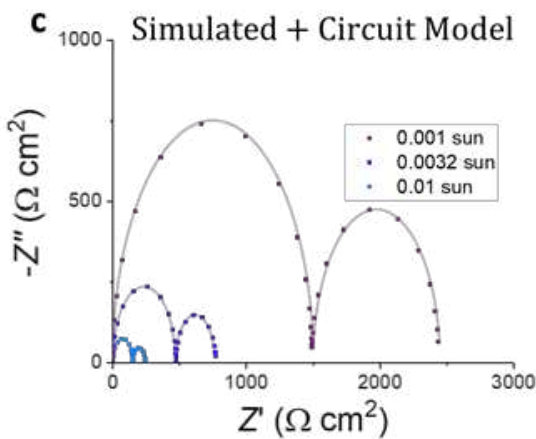

d

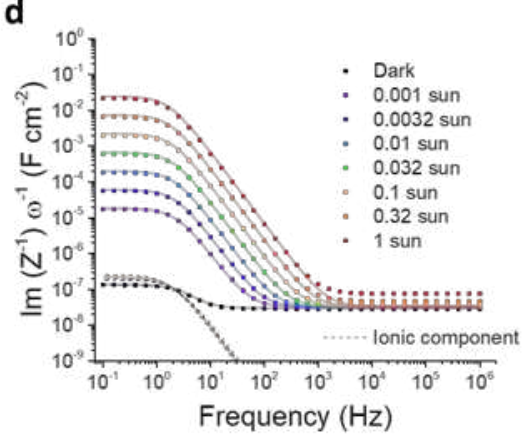

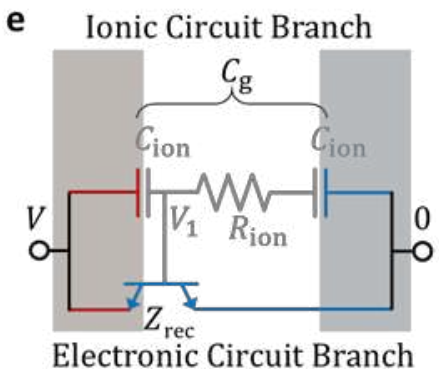

f

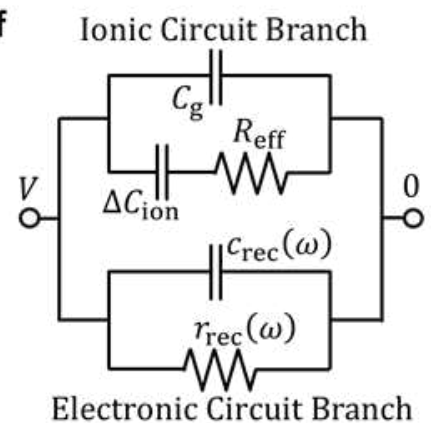


Fig. 2 Measured and simulated impedance spectra of a perovskite solar cell, and transistor-interface recombination circuit model. (a) Nyquist plot of the real ( $\left.Z^{\prime}\right) v s$ imaginary ( $\left.Z^{\prime \prime}\right)$ impedance components, and (b) apparent capacitance, $\omega^{-1} \operatorname{Im}\left(Z^{-1}\right) v s$ frequency of a spiro-OMeTAD/ $\mathrm{Cs}_{0.05} \mathrm{FA}_{0.81} \mathrm{MA}_{0.14} \mathrm{PbI}_{2.55} \mathrm{Br}_{0.45} / \mathrm{TiO}_{2}$ solar cell measured around the open circuit voltage with a perturbation amplitude of $20 \mathrm{mV}$, illuminated with constant bias light intensities (legends of $\mathbf{c}$ and $\mathbf{d}$ respectively, for $V_{\text {OC }}$ values see Table S1, ESI, and for device details see Methods 1.1, ESI). The devices were stabilised to avoid loops in the Nyquist plot arising as artefacts due to incomplete stabilisation of the device during data collection (see Fig. S2, and for characterisation and stabilisation details see Methods 2.2, ESI,). The inset of $\mathbf{b}$ shows the out of phase electronic to ionic current ratio, $j_{\mathrm{rec}}^{\prime \prime} / J_{\text {ion }}$ plotted against half the recombination interface transconductance, $g_{\mathrm{rec}}=\mathrm{d} J_{\mathrm{rec}} / \mathrm{d}\left(V_{1}-V_{\mathrm{n}}\right)$, evaluated from the measurements (Methods 5, ESI). The log-log slope of 1 indicates a linear relationship. (c, d) Corresponding simulated impedance measurements (filled squares) determined from a drift-diffusion model of a p-type/intrinsic/n-type (p-i-n) device structure containing mobile ionic charge. The dashed lines indicate the simulated contribution to the capacitance from mobile ionic charge. (e) The mixed conductor diode circuit model containing an ionically gated transistor used for the simultaneous 5 parameter global fit (continuous lines) to the experimental data (filled squares in $\mathbf{a}$ and $\mathbf{b}$ ) and to the drift-diffusion simulated data (filled squares in $\mathbf{c}$ and $\mathbf{d}$ ). The fit parameters are given in Table S1 (ESI). (f) An alternative representation of the equivalent circuit model shown in e. The elements in the ionic circuit branches are related by $\Delta C_{\text {ion }}=C_{\text {ion }} / 2-C_{\mathrm{g}}$ and $R_{\text {eff }}=R_{\text {ion }}(1+$ $\left.C_{\mathrm{g}} / \Delta C_{\mathrm{ion}}\right)$. The apparent capacitance and recombination resistance elements in the electronic circuit branch, $c_{\mathrm{rec}}(\omega)$ and $r_{\mathrm{rec}}(\omega)$, have a frequency dependence controlled by the ionic circuit branch as derived from the transistor model (see equation 4 and Table 1..

To underpin these measurements with a physical interpretation we simulated impedance spectroscopy measurements using our open source time-dependent drift-diffusion semiconductor model (Driftfusion) which includes the effect of mobile ionic defects ${ }^{26,27}$. The drift-diffusion simulation solves for the time-evolution of free electron, hole, and mobile ionic defect concentration profiles, as well as the electrostatic potential in a $\mathrm{p}-\mathrm{i}-\mathrm{n}$ device in response to illumination and a varying voltage between the terminals as a boundary condition (Methods 3, ESI). In these simulations, we define the dominant recombination mechanism to be via trap states located in the interfacial regions between the p-type hole transporting material (HTM) and the perovskite, and between n-type electron transporting materials (ETM) and the perovskite. The simulation parameters are listed in Table S2, ESI. We have defined the mobility of the ions to be about 11 orders of magnitude lower than the electrons and holes. As a result, the distribution of electrons will maintain a dynamic equilibrium with respect to any changes in electrostatic potential due to ion redistribution. The positive mobile ionic charge is compensated by a uniform distribution of negative static charge, so that the total ionic charge in 
the perovskite is zero mimicking Schottky vacancies where one defect species is mobile. We confine the mobile ionic defects to the perovskite layer. The concentration of mobile ions is defined to be about 12 orders of magnitude greater than the intrinsic electronic carrier concentration in the perovskite so that ionic conductivity is approximately 6 times greater than the intrinsic electronic conductivity of the semiconductor at room temperature in the dark. However, under illumination, or with a forward bias, the increase in electronic charge concentration will result in the electronic conductivity significantly exceeding the ionic conductivity. The qualitative behaviour of the simulations that follow is not sensitive to these numbers as long as: the mean concentration of mobile ionic charge exceeds the mean electronic charge concentration, the bulk electronic conductivity is high enough so that the interfaces dominate the overall electronic impedance, and the bulk electronic conductivity is greater than the ionic conductivity under operation.

Fig. 3a shows an example of the simulated steady state profiles of the conduction band, valence band, and quasi Fermi levels under 1 sun equivalent illumination with an applied d.c. voltage boundary condition $(\bar{V})$ equal to the steady state open circuit voltage $\left(V_{\text {oc }}\right)$. There is no electric field in the bulk of the perovskite layer since the mobile ionic charge has migrated to accumulate at the interfaces screening the built-in potential (Fig. 3a insets) consistent with previous observations and simulations explaining hysteresis. ${ }^{26,28-32}$ Note that, even at 1 sun at open circuit conditions, the majority of the photogenerated electronic charge accumulates in the HTM and ETM at steady state. The amount of electronic charge built up in the perovskite layer is small relative to the amount of mobile ionic charge available to screen changes in potential. Consequently, the changes in electrostatic potential associated with ionic redistribution control the local concentration of electrons and holes in the perovskite. This is important because the concentration of free electrons in the perovskite at the perovskite/HTM interface and the concentration of holes in the perovskite at perovskite/ETM interface determine the rate of recombination via interfacial traps to the respective hole and electron populations in the HTM and ETM layers. Stated another way: the electrostatic potential profile due to ionic charge controls the rate of electron-hole recombination at the interfaces, and this in turn controls current-voltage characteristics of the device. 

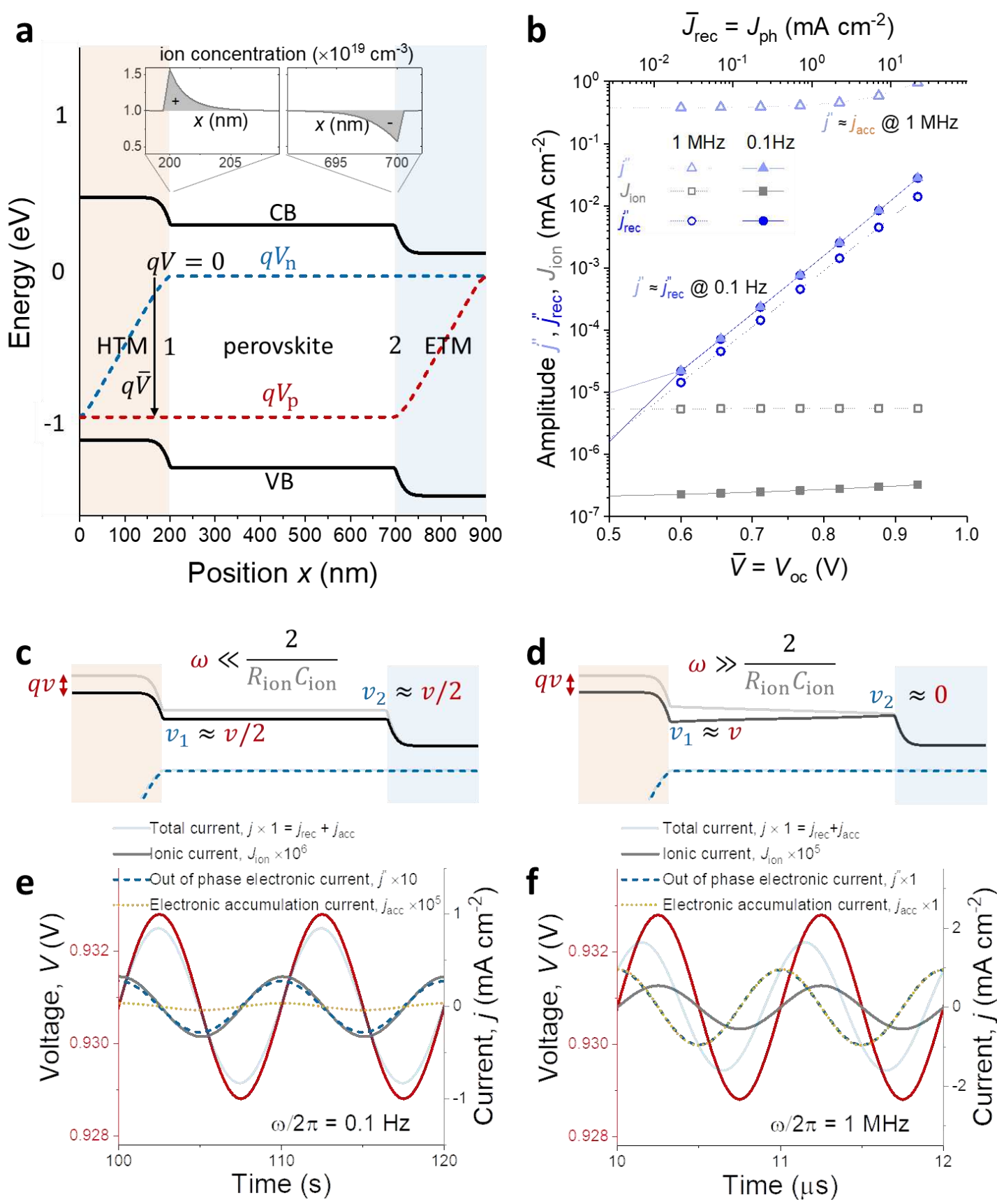

Fig. 3. Drift-diffusion simulations of energy level diagram and ionic/electronic currents during impedance measurements. (a) The steady state electrostatic energy level profile of the conduction band (CB) and valence band (VB) corresponding to the simulations in Fig. $2 \mathrm{c}$ and $\mathrm{d}$ at open circuit under 1 sun equivalent illumination, the insets show net accumulation of ionic charges at the HTM/perovskite and perovskite/ETM interfaces screening the internal electric field. (b) The simulated oscillation amplitudes of the out of phase component of the cell current, $j^{\prime \prime}$, the out of phase component of recombination current, $j_{r e c}^{\prime \prime}$, and the ionic current, $J_{i o n}$, in response to $v$ (with amplitude $\pm 2 \mathrm{mV}$ ) at $1 \mathrm{MHz}$ and $0.1 \mathrm{~Hz}$, plotted against steady state bias voltage $\bar{V}=V_{O C}$ and recombination current $\bar{J}_{r e c}=J_{p h}$. Effect of an applied voltage perturbation with amplitude $v$ superimposed on $\bar{V}=V_{O C}$ (c) at low frequency $(0.1 \mathrm{~Hz})$ 
and (d) at high frequency (1 MHz) on the conduction band energy profile (limits indicated by the black and grey lines). The amplitude of the electrostatic potential oscillations at each interface, $v_{1}$ and $v_{2}$, in response to $v$ are indicated. The corresponding simulated electronic currents (total, $j$, out of phase, $j^{\prime \prime}$, and accumulation, $j_{\text {acc }}$ ) and ionic current $\left(U_{\text {ion }}\right)$ in response to (e) the low frequency and (f) the high frequency applied voltage oscillation $\left(V_{\mathrm{OC}}+v\right.$, red line) vs time. At $1 \mathrm{MHz} j^{\prime \prime} \approx j_{a c c}$, but at $0.1 \mathrm{~Hz} j^{\prime \prime} \approx j_{\text {rec }}$.

We superimposed small oscillating voltages $(v)$ on the applied background bias voltage $(\bar{V})$ boundary condition and simulated the resulting oscillations in current $(j)$ for a range of angular frequencies $(\omega)$ and bias $\bar{V}$. The impedance, $Z(\omega)$, evaluated from these simulations (Fig. 2c and d) shows remarkably similar behaviour to the impedance measurements in Fig. $2 a$ and b. Analysis of the simulations shows that in the dark, with no bias voltage or light, the capacitance of the device, evaluated as $\omega^{-1} \operatorname{Im}\left(Z^{-1}\right)$, is dominated by contributions from the movement of ionic charge accumulating at the interfaces at low frequencies in response to $v$ (dashed lines in Fig. $2 \mathrm{~d})$. However, the exponential increase of $\omega^{-1} \operatorname{Im}\left(Z^{-1}\right)$ at low frequencies when the steady state voltage $\bar{V}$ across the device was increased by light (or applied voltage in the dark, Fig. S2f and g, ESI) does not arise directly from the ions, and is also not due to an accumulation of electronic charge (see Fig. S2j-l, ESI and the magnitude of electronic accumulation current in Fig. 3e). Instead, this apparent capacitance arises from current due to the out of phase modulation of electronic recombination at the interfaces. This explanation of the apparent capacitance is consistent with the interpretation of Jacobs et al. developed in independently of this study. ${ }^{33}$

The explanation is seen in Figs $3 c$ and $d$ which show that ionic redistribution influences the electrostatic potential profile dropping across the perovskite layer when the applied voltage perturbation $(v)$ oscillates at sufficiently low frequencies for the ions to move. The electronic carrier concentration profile responds to form a dynamic equilibrium with the changing electrostatic potential due to mobile ions. At low frequency, the voltage screening effect of ionic redistribution (with associated capacitive ionic current $J_{\text {ion}}$ ) occurs out of phase with $v$ resulting in out of phase modulation of the interfacial recombination of electronic charge $\left(j_{\text {rec }}^{\prime \prime}\right)$, and thus current through the device $\left(j^{\prime \prime} \approx j_{\text {rec }}^{\prime \prime}\right.$, Fig. $3 \mathrm{e}$ and $\left.\mathrm{b}\right)$. At high frequencies the ionic redistribution is too slow for ions to compensate the rapid changes in applied potential, so recombination only varies in phase with $v$; in this case the out of phase current component arises primarily from capacitive accumulation of electronic charge in the HTM and ETM contacts $j^{\prime \prime} \approx j_{\text {acc }}$, Fig. $3 \mathrm{f}$ and b).

The changes in electrostatic potential due to the oscillation of ionic charge at low frequencies can be viewed as varying the magnitude of the barrier to charge transfer through the device from each direction. This interfacial charge transfer is mediated by the processes of interfacial recombination and thermal generation similar to a standard diode. However, it is as if the builtin potential barrier of the diode junction is being modulated in addition to the voltage being applied across it (c.f. Fig. 1a and 1c). 'Barrier' in this context refers to the energy that would be 
required to promote an electron (or hole) from the quasi Fermi energy on either side of the interface to the conduction (or valence) band of the perovskite at the interface (Fig. 1c). The local electrostatic potential, which arises from the solution of Poisson's equation accounting for the profile of ionic charge (in addition to the electrons and holes), thus varies this 'barrier' and determines the local change in concentration of electrons and holes by the corresponding Boltzmann factor $\left(\exp \left[q v_{1} / k_{\mathrm{B}} T\right]\right.$ and $\exp \left[-q v_{1} / k_{\mathrm{B}} T\right]$ respectively at interface 1 , Fig. 3c) when the system is in a dynamic equilibrium. This in turn controls the charge transfer rate of recombination and generation.

\section{Ionically gated interfacial transistor}

We now develop simple expressions to describe the characteristics and impedance of the interfaces in a semiconductor with mixed ionic and electronic conduction by considering how the current across each interface will vary with the externally applied voltage in the presence of inert mobile ions. We will show that these expressions, represented by the circuit model shown in Fig. 2e (or Fig. 4g or $\mathrm{h}$ for more complete descriptions), give an excellent approximation to the results of the ionically coupled drift-diffusion simulations described above. This allows the time or frequency dependent behaviour of hybrid perovskite solar cells in response to changing biases to be easily evaluated.

In these devices, the interfacial electronic currents can be related to the processes of charge injection, collection, thermal generation and recombination between the active semiconductor layer and the hole transporting material (HTM) or electron transporting material (ETM) layers. The currents related to these processes are indicated in Fig. 4a. Under most circumstances one of these processes will dominate the impedance of the device, either for the free electron or free hole species (c.f. Note S6, ESI). We assume resistance to free electron and hole transport in the perovskite is low relative to the recombination/generation and the injection/collection impedances, consistent with measurements showing long diffusion lengths observed in these materials ${ }^{34,35}$. We also assume that the influence of ionic defect accumulation on the recombination rate constant is of secondary importance relative to the electrostatic effect of the ions, although it could have an influence in some cases ${ }^{36}$. In cases where interfacial recombination centres are passivated, photogenerated charge can accumulate in the perovskite layer and 'screen' hysteresis ${ }^{26,29}$. This passivation could be modulated by a varying concentration of ionic defects, but we neglect any such effects here. 

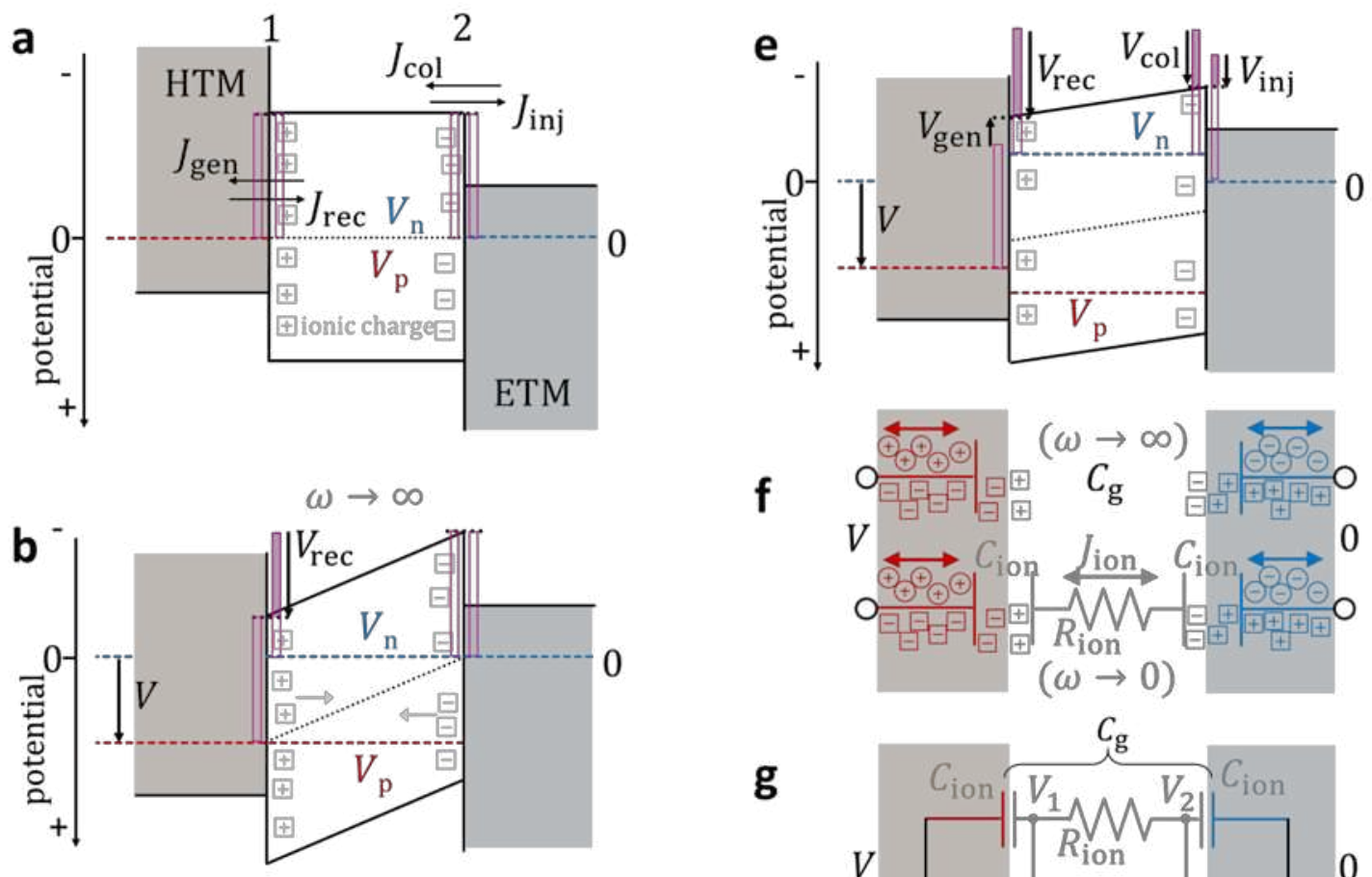

f

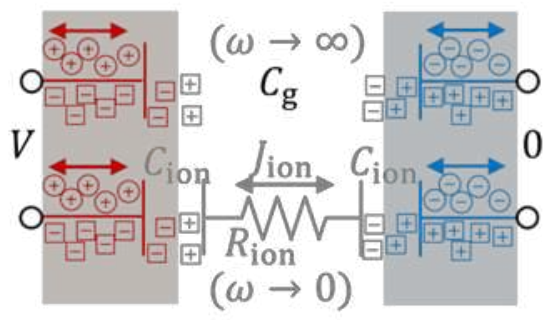

g
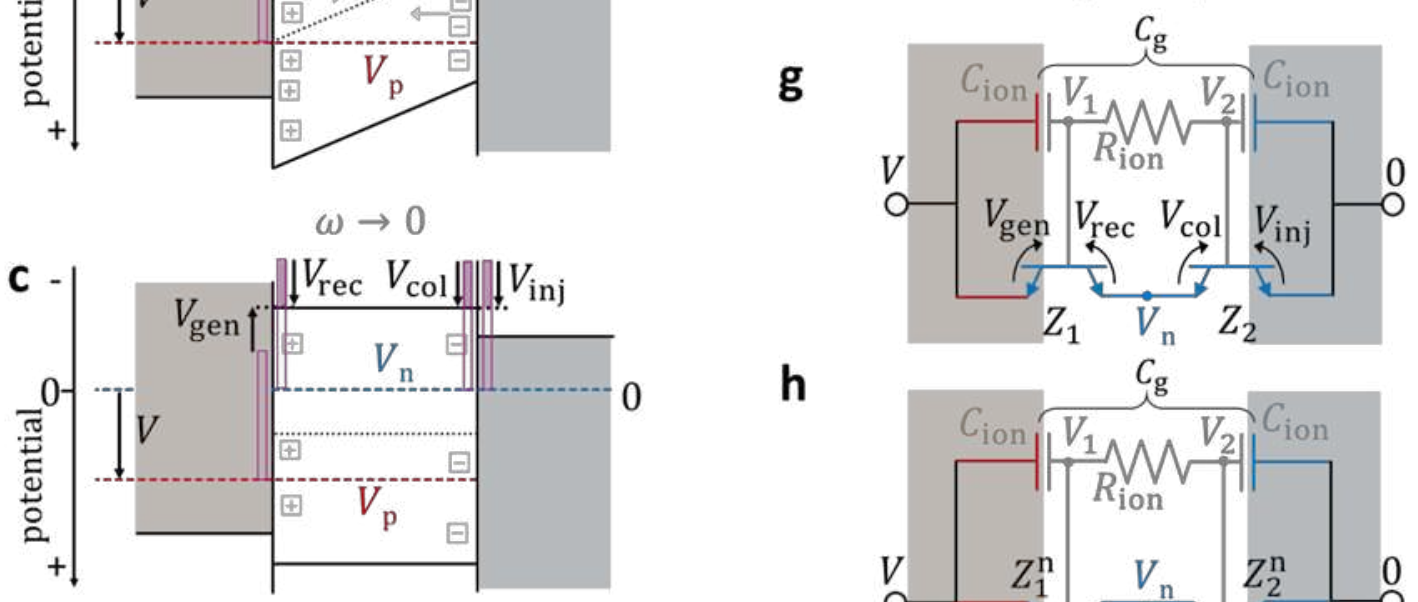

h

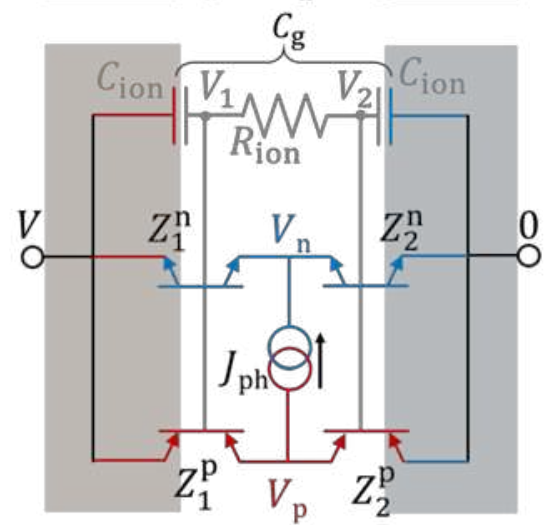

$1 \mathrm{HTM} /$ perovskite interface

2 perovskite/ETM interface

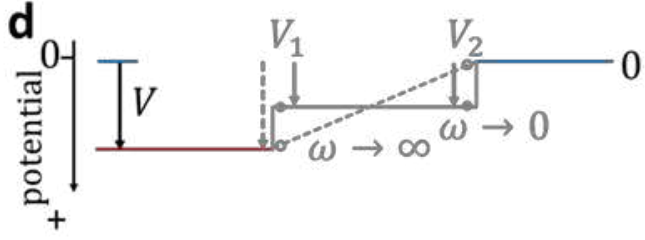

cell potential

$V_{n}$ electron quasi Fermi level

$V_{\mathrm{p}}$ hole quasi Fermi level

$V_{\text {gen }}$ change in generation potential

$V_{\text {rec }}$ change in recombination potential

$J_{\text {gen }}$ thermal generation current density

$J_{\text {rec }}$ recombination current density

$J_{\text {col }}$ collection current density

$V_{\text {col }}$ change in collection potential

$V_{\text {inj }}$ change in injection potential

$\omega$ angular frequency of perturbation

equilibrium barrier potential

$J_{\text {ion }}$ ionic current density

$J_{\text {inj }}$ injection current density

$R_{\text {ion }}$ ionic resistance

$C_{\text {ion }}$ interfacial space charge capacitance

$J_{\mathrm{ph}}$ photogeneration current density

$C_{\mathrm{g}}$ geometric capacitance of cell

change in electrostatic potential at 2

$Z_{1}^{\mathrm{n}}\left(Z_{1}^{\mathrm{p}}\right)$ interface 1 electron (hole) impedance

Fig. 4 Simplified energy level diagrams and circuit models using transistors to describe the ionic gating of electron processes at different interfaces. The dark equilibrium barrier height is indicated by the unfilled purple rectangles. In non-equilibrium situations, a reduction in barrier height is indicated by the filled section. (a) The energy levels of the conduction and valence bands in the dark after equilibration of ionic charge. Due to detailed balance the interfacial currents are equal and opposite $U_{\text {gen }}=J_{\text {rec }}=J_{\text {s1 }}$ at interface 1 and $J_{\mathrm{col}}=J_{\mathrm{inj}}=J_{\mathrm{s} 2}$ at interface 2 ) at dark equilibrium. The corresponding energy level profiles after applying a voltage $V$, in the dark $\left(V_{\mathrm{n}}=0\right)$, for a device whose 
impedance is limited by electron recombination (b) immediately after the voltage is applied $(\omega \rightarrow \infty)$ and (c) after the redistribution of ionic charge has reached steady state $(\omega \rightarrow 0)$. The changes in barrier heights $\left(V_{\text {gen }}, V_{\text {rec }}, V_{\text {col }}\right.$ and $\left.V_{\text {inj }}\right)$ for the various interfacial electron transfer processes in response to an applied potential $V$ and the electron quasiFermi potential $\left(V_{\mathrm{n}}\right)$ are indicated. (d) The corresponding change in the electrostatic potential profile (dashed line - instantaneous, solid line - steady state). The changes in electrostatic potential at interfaces 1 and 2 are indicated by $V_{1}$ and $V_{2}$. The relationship between these changes is given in Table 1. (e) A general example for a device in the light (where the electron quasi Fermi level $V_{\mathrm{n}} \neq 0$ ). In this case the device impedance has contributions from both interfaces and the ions have not reached a steady state distribution. (f) The equivalent circuit model for the impedance of the ionic circuit branch in response to high frequency voltage perturbation, $v(\omega \rightarrow \infty)$, where perovskite ions are effectively frozen, and at lower frequencies, $v(\omega<\infty)$ where perovskite ionic motion is described by $C_{\text {ion }}-R_{\text {ion }}-C_{\text {ion }}$ series elements. Here we assume the dopant ions in the HTM and ETM (red and blue squares) are static. (g) An equivalent circuit model for the device in which the impedance to electron transfer for both interfaces are modelled as bipolar transistors with impedance $Z_{1}$ and $Z_{2}$, the base terminals are gated by the ionic potentials $V_{1}$ and $V_{2}$. The curved arrows indicate the potential differences between the 'terminals' on the transistor elements. (h) General circuit model considering both electrons ( $\mathrm{n}$ ) and holes ( $\mathrm{p}$ ) with a (negative) photogeneration current $\left(U_{\mathrm{ph}}\right)$, where the potential of the electrons $\left(V_{\mathrm{n}}\right)$ and holes $\left(V_{\mathrm{p}}\right)$ in the perovskite layer correspond to the electron and hole quasi Fermi levels.

Initially we consider the impedance related to the recombination (and thermal generation) of electrons at the interface with p-type HTM (interface 1) assuming electron injection and collection is not limiting. Close to the interface, where most recombination is thought to occur ${ }^{37}$, 38 , electrons in the perovskite phase with concentration $n_{1}$ may be considered a minority species relative to the holes in the neighbouring HTM. Here, for simplicity we assume the electron recombination current density from the perovskite to HTM can be approximated by the first order process, $J_{\text {rec }} \propto n_{1}$. For the fits to data described later we explicitly account for the ideality factor of the interfaces, allowing for non-linear recombination, see Methods 4.2 (ESI).

The recombination current density $J_{\text {rec }}$ varies exponentially with the potential 'barrier' given by the difference between the conduction band edge of the perovskite at interface 1 and electron quasi Fermi level in the perovskite $\left(V_{\mathrm{n}}\right)$ which we reference to the ETM Fermi level $(0 \mathrm{~V})$. At dynamic equilibrium, this barrier height controls the population of free electrons in the perovskite available to recombine at the interface by the corresponding Boltzmann factor (see Fig. 1c). In addition to $J_{\text {rec, }}$ there will also be a thermal generation current, $-J_{\text {gen }}$, of electrons from the HTM to the perovskite. This current density, in the opposite direction to $J_{\text {rec }}$ across the interface, varies exponentially with the potential barrier given by the difference between the conduction band of the perovskite at interface 1 and the Fermi level in the HTM $(V$, the cell bias 
voltage - since the Fermi level of the ETM is defined to be zero). Similarly, under dynamic equilibrium conditions, this barrier height determines the population of electrons in the HTM at the perovskite interface with sufficient energy to be promoted to the perovskite conduction band from the HTM. At dark equilibrium $(V=0)$ the barrier for the two processes is the same (see the open pink bars on either side of the interface in Fig. 4a and no bias case of Fig. 1c). Since the system must obey the principle of detailed balance at equilibrium, there will be equal and opposite current densities across the barrier with magnitude $J_{\mathrm{rec}}=-J_{g e n}=J_{s 1}$. Here, $J_{\mathrm{s} 1}$ is the saturation current density of recombination for interface 1.

We refer to the changes in the potential barrier relative to the dark equilibrium case for the recombination and the generation current as $V_{\text {rec }}$ and $V_{\text {gen }}$ respectively (at dark equilibrium $V_{\text {rec }}=V_{\text {gen }}=0$ ). The net electron recombination at this interface is then given by:

$J_{1}=J_{\mathrm{rec}}-J_{\mathrm{gen}}=J_{\mathrm{s} 1} e^{\frac{q V_{\mathrm{rec}}}{k_{\mathrm{B}} T}}-J_{\mathrm{s} 1} e^{\frac{q V_{\mathrm{gen}}}{k_{\mathrm{B}} T}}$

where $q$ is the electronic charge, $k_{\mathrm{B}}$ is Boltzmann's constant, and $T$ is temperature (see Fig. 4 and Fig. S4, ESI). Without mobile ions in the system, a potential, $V$, applied across the cell would be fully experienced by the electrons in the perovskite at interface 1 so that $V_{\text {rec }}=V$ with no change in the barrier to thermal generation $\left(V_{\text {gen }}=0\right)$ so equation 1 would become the standard diode equation: $J_{1}=J_{\mathrm{s} 1}\left(\exp \left[q V / k_{\mathrm{B}} T\right]-1\right)$.

However, as observed in the simulations, the electrostatic potential at the interfaces in hybrid perovskites devices depend both on the applied potential $V$ and also on the effect of the redistribution of mobile ions. Ionic redistribution modifies the electrostatic potential and thus the barrier height at the HTM perovskite interface. This influences the values of both $V_{\text {rec }}$ and $V_{\text {gen }}$ as illustrated in Figs $4 \mathrm{~b}$-e. Here, we refer to the changes in the electrostatic potential at the interfaces 1 and 2 relative to the values at dark equilibrium as $V_{1}$ and $V_{2}$ (as indicated schematically in Fig. 4d). The relationships between these various changes in potential is expressed in Table 1 and will be discussed below.

\section{Table 1 Expressions for potentials driving electron transfer processes, and circuit} branch impedances. The terms in the equations are illustrated in Fig. 4 and defined in the text. *The impedance for the electronic branch of the circuit is given for the specific case where impedance due to recombination of a single carrier dominates (more general cases are discussed in the Methods, Notes S2 and S6, and Tables S3 and S4, ESI). The impedance of the electronic circuit branch, $Z_{\text {rec }}$, is given in terms of the apparent capacitance and resistance of the interface $c_{\text {rec }}$ and $r_{\text {rec }}$ which are represented in Fig. 2f. 


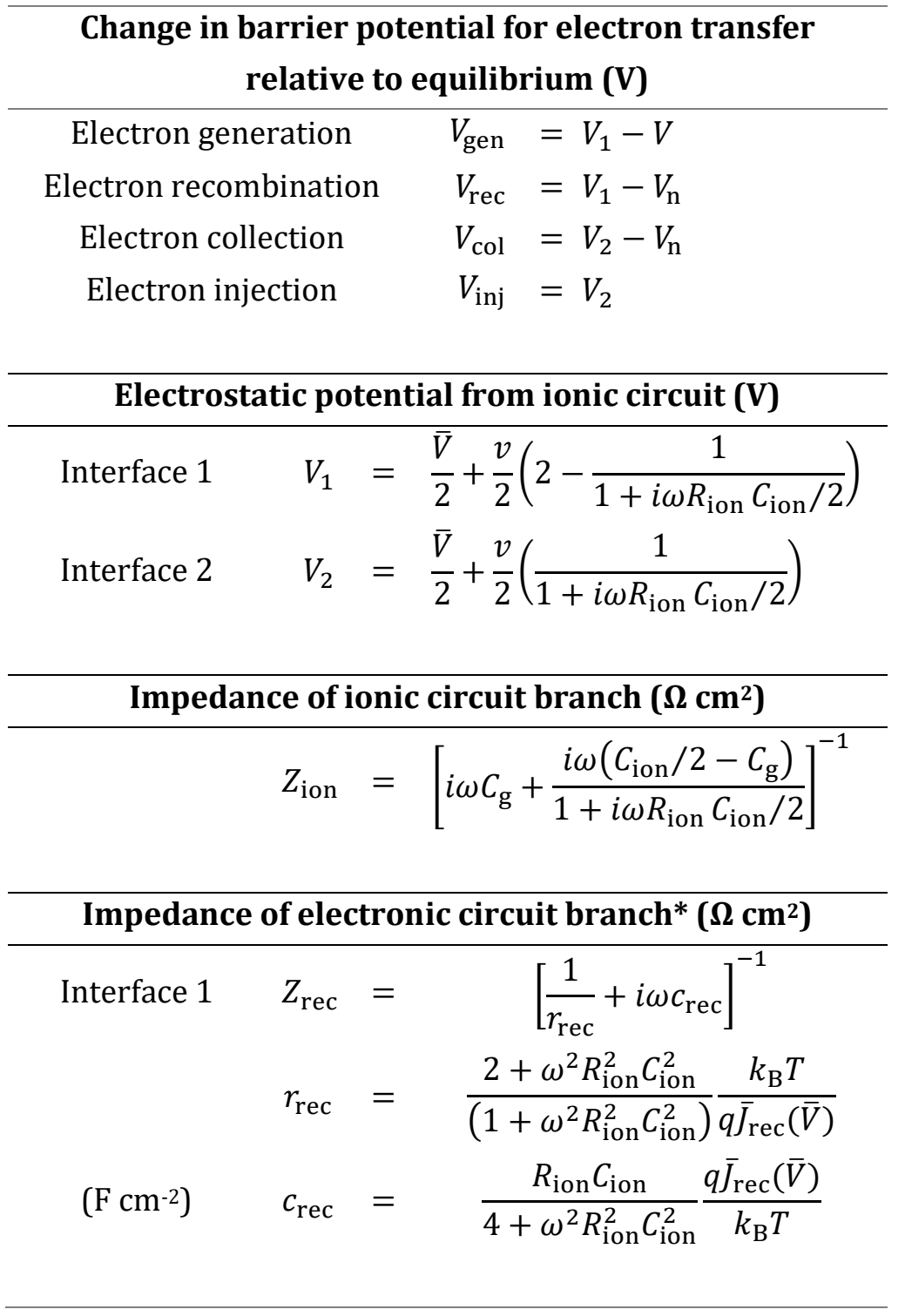

In the simple case of a p-i-n device with ion blocking contacts and symmetric capacitances at each contact, ion redistribution occurs with a time constant approximated by $R_{\mathrm{ion}} C_{\mathrm{ion}} / 2$. $R_{\mathrm{ion}}$ is the specific resistance $\left(\Omega \mathrm{cm}^{2}\right)$ to ionic motion across the perovskite layer. $C_{\text {ion }}$ is the specific capacitance $\left(\mathrm{F} \mathrm{cm}^{-2}\right)$ of the interfacial space charge layer corresponding to the accumulation of mobile ionic defects in the perovskite and uncompensated static dopant ions in the HTM or ETM (Fig. 4f). If the concentration of mobile ionic defects is large relative to the concentration of free electrons and holes in the active layer then the ionic distribution will determine the electrostatic potential profile in the perovskite layer. The change in electrostatic potential at each interface, $V_{1}$ and $V_{2}$, can be found by analysing the voltage drop on either side of the resistor in the $C_{\text {ion- }}{ }^{-}$ $R_{\text {ion }}-C_{\text {ion }}$ series when a voltage $V=\bar{V}+v(\omega)$ is applied across the whole series Table 1. This is because $V$ is given by the sum of the voltages across each element: $V=V_{C \text { ion }}+V_{R \text { ion }}+V_{C \text { ion }}$ so that $V_{1}=V-V_{C \text { ion }}$ (and $V_{2}=V_{C \text { ion }}$ ). At steady state the ionic current density, $J_{\text {ion, }}$ is zero so that $V_{R \text { ion }}=J_{\text {ion }} R_{\text {ion }}=0$ and $\bar{V}_{1}=\bar{V}_{2}=V_{C \text { ion }}=\bar{V} / 2$ in this simple case where both capacitances are equal. In the frequency domain for the small perturbation, we can also state $v(\omega)=$ $J_{\text {ion }} / i \omega C_{\text {ion }}+J_{\text {ion }} R_{\text {ion }}+J_{\text {ion }} / i \omega C_{\text {ion }}$ so that rearranging for $J_{\text {ion }}$ and substituting into $v_{1}=v-$ 
$J_{\text {ion }} / i \omega C_{\text {ion }}$ (and $v_{2}=J_{\text {ion }} / i \omega C_{\text {ion }}$ ) yields $V_{1}=\bar{V}_{1}+v_{1}$ (and $V_{2}=\bar{V}_{2}+v_{2}$ ) as given in Table 1 . We treat cases with different capacitances at each interface in Note S6 and Table S4 (ESI).

This description assumes that changes in electrostatic potential across the interfaces due to ionic accumulation predominantly drop within each contact material (as sketched in Fig. 1c and simulated in Fig. 3a). This will be the case when the mobile ionic defect concentration is significantly greater than the doping concentration of the contact materials. We discuss the case where there is a significant drop in electrostatic potential in the perovskite (depicted in Figure S1a, ESI) as well as including the dependence of $C_{\text {ion }}$ on $\bar{V}$ due to variation in the space charge layer widths in the contacts in the Methods section S4 (ESI).

Based on these assumptions, equation 1 gives a general expression for the net electron recombination current across interface 1 in terms of the applied potential $V$, the electron quasi Fermi level $V_{\mathrm{n}}$, and the change in electrostatic potential of the interface $V_{1}$ by substituting them into the expressions for $V_{\text {gen }}$ and $V_{\text {rec }}$ (Table 1):

$J_{1}=J_{\mathrm{s} 1}\left[e^{\frac{q\left(V_{1}-V_{\mathrm{n}}\right)}{k_{\mathrm{B}} T}}-e^{\frac{q\left(V_{1}-V\right)}{k_{\mathrm{B}} T}}\right]$

This is analogous to the expression used to describe a bipolar n-p-n transistor (c.f. Figs $1 \mathrm{~b}$ and c where $V_{\mathrm{E}}$ and $V_{\mathrm{n}}$ are zero) where the electrostatic potential of interface 1 behaves like the transistor base (so the device only has two 'external' terminals). The voltage of this conceptual base, $V_{1}$, relative to the Fermi level of the ETM/cathode $(0 \mathrm{~V})$, arises from any change in ionic accumulation at interface 1 . Under dark forward bias conditions $(V>0)$ there is net flux of electrons from the perovskite (which acts as the emitter with potential $V_{\text {n }}$ ) to the HTM (which acts as the collector with potential $V)$. The potential differences of the base-emitter $\left(V_{\mathrm{BE}}=V_{\mathrm{B}}-\right.$ $\left.V_{\mathrm{E}}\right)$ and base-collector $\left(V_{\mathrm{BC}}=V_{\mathrm{B}}-V_{\mathrm{C}}\right)$ junctions are equivalent to $V_{\text {rec }}$ and $V_{\text {gen }}$ respectively (c.f. Fig. 1b). We have modified the conventional bipolar transistor symbol to emphasise that the net electronic current through the transistor may be in either direction according to the electrical and light bias conditions. If $V_{\text {rec }}<V_{\text {gen }}$ (e.g. under reverse bias), then the conventional assignment of the terms 'emitter' and 'collector' to the two sides of the interface would be reversed. If there is no chemical reaction between ionic and electronic charge at the interface and no ionic penetration into the HTM, then the ionic-to-electronic current gain of the

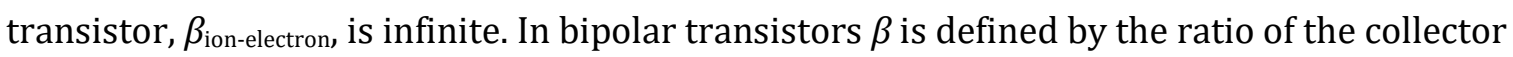
current to the base current. In this basic case, only electronic charge may be transferred across the interface (collector current $=J_{1}$ ) and ionic charge is confined to the perovskite and cannot cross the interface (base current $=0$ ) despite the possibility of an ionic current, $J_{\text {ion }}$, in the rest of the perovskite. We note that a field effect transistor with an infinite insulator capacitance operating in the subthreshold regime would also display similar properties. 
These observations naturally result in the simple equivalent circuit illustrated in Fig. 1 f and 2e where an 'ionic circuit' branch is connected in parallel to an 'electronic circuit' branch. The complex impedance of the ionic branch of the circuit $\left(Z_{\text {ion }}\right)$ behaves analogously to an insulating material which shows dielectric loss through Debye relaxation of charge (in this case ionic polarisation) with an equivalent series resistance corresponding to the $R_{\text {ion. }}$ At high frequencies, when the ionic charge is effectively static, $Z_{\text {ion }}$ is dominated by the device's geometric capacitance $\left(C_{\mathrm{g}}\right)$ but at lower frequencies the ionic motion in the $C_{\mathrm{ion}}-R_{\mathrm{ion}}-C_{\text {ion }}$ series dominates (Fig. 4f). There is a continuous transition between these two regimes at a characteristic angular frequency $2 /\left(R_{\mathrm{ion}} C_{\mathrm{ion}}\right)$. This results in the expression for the ionic impedance presented in Table 1 which is derived in Methods 4.1 (ESI). The expression for $Z_{\text {ion }}$ describes the transition between the frequency regimes using physically meaningful quantities. The frequency dependent contribution from $C_{\mathrm{g}}$, which is electronic but has been included in the expression for $Z_{\text {ion, }}$ is represented in the equivalent circuit by a curly bracket across the ionic branch. Note that this is different to connecting $C_{\mathrm{g}}$ in parallel to the ionic elements which would yield an unphysical expression for $Z_{\text {ion }}$. As discussed, the $C_{\text {ion }}-R_{\text {ion }}-C_{\text {ion }}$ series components enable the straightforward evaluation of $V_{1}$ (and $V_{2}$ ) in terms of $V$ (these are given in Table 1). The change in electrostatic potential due to ion redistribution, $V_{1}$, controls the base of transistor element and consequently the impedance of the electronic branch of the equivalent circuit. This will be discussed in detail further below. Strikingly, virtually all the features related to the electronic behaviour of a perovskite solar cell, under the conditions described above, can be summarised through the use of this single circuit element coupling the electrostatic potential due to ions to electronic charge transfer, i.e. a transistor.

An alternative, more conventional equivalent circuit, representation of this same system is shown in Fig. 2f, however, the physical meaning of the elements is less intuitive. In the ionic circuit branch $\Delta C_{\text {ion }}=C_{\text {ion }} / 2-C_{\mathrm{g}}$ and $R_{\text {eff }}=R_{\text {ion }}\left(1+C_{\mathrm{g}} / \Delta C_{\text {ion }}\right)$ as discussed in Methods 4.1 (ESI). The apparent capacitance and recombination resistance elements in the electronic circuit branch, $c_{\text {rec }}(\omega)$ and $r_{\text {rec }}(\omega)$, have a frequency dependence controlled by the ionic circuit branch as derived from the transistor model discussed in the following sections (the expressions for them are given in Table 1. We now consider the implications of a transistor-like interface for the behaviour of the device.

\section{Ionic-to-electronic current amplification}

Amplification is a key property shown by bipolar transistors ${ }^{21}$, where changes in electronic energy barriers induced by the gating terminal (base) amplify the flux of electrons or holes between the emitter and collector terminals. The simulated impedance spectroscopy results show that, at sufficiently low frequency voltage oscillations, the out of phase component of the electronic current oscillations is directly proportional to the ionic current in the device (c.f. solid grey and dashed blue curves in Fig. 3e). The amplitude of this out of phase electronic current scales in direct proportion to the steady state current $\bar{J}_{\text {rec }}$ across the interface (see Fig. $3 \mathrm{~b}$ where $\bar{J}_{\text {rec }}=J_{\text {ph }}$, the photogenerated current, since each simulation is around $V_{\mathrm{oc}}$ ). This implies that 
there is an ionic-to-electronic current amplification process that can occur in mixed conducting devices such as perovskite solar cells and the effect varies exponentially in magnitude with the steady state bias voltage dropping across the interface. We now examine the mechanism underlying this effect.

At low frequencies when $\omega \ll 2 /\left(R_{\text {ion }} C_{\text {ion }}\right)$ the impedance due to $R_{\text {ion }}$ becomes negligible relative to that of the $C_{\text {ion }}$ elements in series so that the ionic current can be approximated by $J_{\text {ion }}=v / Z_{\text {ion }} \approx i \omega C_{\text {ion }} v / 2$. This ionic current induces an out of phase change in potential at the interface of $v_{1}^{\prime \prime}=J_{\text {ion }} R_{\text {ion }} / 2$ due to the potential dropped across $R_{\text {ion }}$ with the small ionic current $J_{\text {ion }}$ flowing through the perovskite. Since oscillations in the potential controlling recombination rate are equal to the changes in potential at the interface, $v_{\text {rec }}=v-v_{\mathrm{n}}=v_{1}$ (since the potential of the electrons in the perovskite is pinned to the potential of the ETM, i.e. $v_{\mathrm{n}}=V_{\mathrm{n}}=0$ ), there will be an out of phase component to the electronic current given by $j_{\mathrm{rec}}^{\prime \prime}=v_{\mathrm{rec}}^{\prime \prime} g_{\mathrm{rec}}=J_{\mathrm{ion}} R_{\mathrm{ion}} g_{\mathrm{rec}} / 2$. Here $g_{\text {rec }}$ is the recombination transconductance which describes the change in interfacial current in response to changes in $V_{\text {rec }}$ given by $g_{\text {rec }}(\bar{V})=\mathrm{d} J_{\text {rec }} / \mathrm{d}\left(V_{1}-V_{\mathrm{n}}\right)=q \bar{J}_{\text {rec }} / k_{\mathrm{B}} T$, where $V_{\mathrm{n}}=0 \mathrm{~V}$ in this example. Taking the ratio between the out of phase electronic and ionic currents gives an ionic-to-electronic transcarrier amplification factor:

$\frac{j_{\mathrm{rec}}^{\prime \prime}}{J_{\text {ion }}}=\frac{R_{\text {ion }}}{2} g_{\text {rec }}(\bar{V})=\frac{R_{\text {ion }}}{2} \frac{q \bar{J}_{\text {rec }}(\bar{V})}{k_{\mathrm{B}} T}$

analogous to the classic result for an amplification circuit using a bipolar transistor. The magnitude of $j_{\text {rec }}^{\prime \prime}$ across the interface is proportional to $R_{\text {ion }}$, independent of the value of $C_{\text {ion }}$, and will also increase exponentially with background bias voltage, $\bar{V}$. For large voltage perturbations $g_{\text {rec }}$ will no longer be constant during the perturbation.

Rearranging equation 3 gives $R_{\text {ion }}=2 j_{\text {rec }}^{\prime \prime} /\left(J_{\text {ion }} g_{\text {rec }}\right)$. Interestingly, this result implies that $R_{\text {ion }}$ (and thus ionic conductivity) can be inferred from measurements of the device's apparent capacitance $\left(c_{\mathrm{rec}}\right)$ due to modulated electron recombination. This is because both the out of phase electronic recombination current, $j_{\text {rec }}^{\prime \prime}$, and the ionic current, $J_{\text {ion }}$ (as $\omega \rightarrow 0$ ), are directly proportional to the measured capacitance of the device so that $j_{\text {rec }}^{\prime \prime} / J_{\text {ion }}=2 c_{\text {rec }}(\bar{V}) / C_{\text {ion. }}$. The meaning of $c_{\text {rec }}$ which results in this relationship is discussed further in the next section. Experimentally, $g_{\text {rec }}(\bar{V})$ can be found if $\bar{J}_{\text {rec }}$ can be estimated from the measured data (see Methods 5, ESI). $C_{\text {ion }}$ can easily be determined from the measurements of the low frequency device capacitance in dark conditions with $\bar{V}=0 \mathrm{~V}$ and $c_{\text {rec }}$ determined from the apparent capacitance with a bias voltage $\bar{V}$ (in the light or dark). The inset of Fig. $2 \mathrm{~b}$ shows that this method predicts a value of $R_{\mathrm{ion}} \approx 60 \mathrm{k} \Omega \mathrm{cm}^{2}$ (ionic conductivity of about $10^{-9} \mathrm{~S} \mathrm{~cm}^{-1}$ ) for the cell under consideration.

\section{Capacitor-like and inductor-like behaviour}

The ionic gating effect at the interfaces results in out of phase electronic currents causing the device to display very large apparent capacitances or inductances at low frequencies. We now 
explore the implications of this. Under forward bias $(V>0)$ conditions $J_{\text {rec }} \gg J_{\text {gen }}$ so the second term of equation 2 can be neglected such that $J_{1} \approx J_{\text {rec }}=J_{\mathrm{s} 1} \exp \left[q V_{1} / k_{\mathrm{B}} T\right]$ when $V_{\mathrm{n}}=0$. We see that the electronic current through the device is controlled by an expression similar to a diode, but with an electrostatic potential $V_{1}$ instead of a voltage $V$ across the terminals. The expression for $V_{1}\left(V, R_{\text {ion }}, C_{\text {ion }}, \omega\right)$ is given in Table 1 , substituting it in, and differentiating $J_{\text {rec }}$ with respect to the applied voltage $V$ gives an expression for the reciprocal of the recombination impedance, which in the small voltage perturbation $(v)$ limit can be written:

$\frac{1}{Z_{\mathrm{rec}}(\bar{V})}=\frac{\mathrm{d} j_{\mathrm{rec}}}{\mathrm{d} v}=\frac{1}{2}\left(2-\frac{1}{1+i \omega R_{\mathrm{ion}} C_{\mathrm{ion}} / 2}\right) \frac{q}{k_{\mathrm{B}} T} \bar{J}_{\mathrm{rec}}(\bar{V})=\frac{1}{r_{\text {rec }}}+i \omega c_{\mathrm{rec}}$

where the background recombination current across the interface with a potential difference $\bar{V}$ at steady state $(\omega=0)$ is $\bar{J}_{\text {rec }}(\bar{V})=J_{\mathrm{s} 1} \exp \left[q \bar{V} /\left(2 k_{\mathrm{B}} T\right)\right]$. Separating $1 / Z_{\text {rec }}$ into its real and imaginary parts enables expressions for the small perturbation recombination resistance of the interface, $r_{\text {rec }}$, and the apparent electronic capacitance of the interface, $c_{\text {rec }}$ to be determined in terms of $R_{\text {ion }}, C_{\text {ion }}$ and $\omega$ (these expressions are written out in Table 1 . Several features of $r_{\text {rec }}$ and $c_{\text {rec }}$ are noteworthy. First, $r_{\text {rec }}$ shows a dependence on frequency since the amplitude of the interfacial barrier $\left(v_{1}-v_{\mathrm{n}}\right)$ oscillations is frequency dependent so that $r_{\text {rec }}(\omega \rightarrow 0)=2 r_{\text {rec }}(\omega \rightarrow$ $\infty)$. Second, the interface behaves like a frequency dependent capacitor despite no accumulation of electronic charge being required; using the expression for $c_{\text {rec }}$ in Tabl 1 and $g_{\text {rec }}(\bar{V})=q \bar{J}_{\text {rec }}(\bar{V}) / k_{\mathrm{B}} T$ from equation 3 we see that at low frequency $c_{\text {rec }}(\omega \rightarrow 0)=$ $R_{\text {ion }} C_{\text {ion }} g_{\text {rec }} / 4$ but at high frequency $c_{\text {rec }}(\omega \rightarrow \infty)=0$. Third, the magnitude of $c_{\text {rec }}$ is proportional to $\bar{J}_{\text {rec }}(\bar{V})$ and so increases exponentially with the voltage (which may be photoinduced) across the interface allowing variable control of the apparent capacitance. This capacitive behaviour could not be used for energy storage, since it arises from the modulation of a large background current flowing across the interface. However, the effect offers a route to achieve at least $10^{3}$ times greater effective capacitance per unit area at low frequency than the capacitance achieved by state-of-the-art hafnium oxide capacitors used in electronic circuitry $\left(\sim 2 \times 10^{-6} \mathrm{~F} \mathrm{~cm}^{-2}\right)^{39}$.

Global fits to both experimental measurements and drift-diffusion simulated measurements are shown in Fig. 2 using the expression for $Z_{\text {rec }}$ based on equation 4 incorporated in the circuit model shown in Fig. 1 . Only five free parameters are required to simultaneously fit all measurement conditions. The complete equation for the circuit model fit to the data is given in the Methods 4.3 (ESI). The inputs to the fitting model are: the measured impedance spectra, $Z(\omega)$; the bias voltages ( $\bar{V}=V_{\text {OC }}$, for open circuit measurements) at which these were collected; and the steady state ideality factor, $m_{\mathrm{ss}}$, determined from the $V_{\mathrm{OC}} v s$ light intensity relationship of the device. The free fitting parameters in the model are: $C_{\mathrm{g}}, R_{\mathrm{ion}}, C_{\mathrm{ion}}, J_{\mathrm{s} 1}$, and $f_{\mathrm{c}}$, the fraction of interfacial electrostatic potential dropping within the contacts (Table S1, ESI, presents the values of the fitting parameters). Since we define the current gain of the transistor $\left(\beta_{\text {ion-electron }}\right)$ to be infinite, the transistor element is described by only two parameters, $J_{s 1}$, and its ideality factor, 
$m_{1}$, which is related to $m_{\mathrm{ss}}$ and $f_{\mathrm{c}}$ (a more detailed explanation of $f_{\mathrm{c}}$ and $m_{1}$ is given in Methods 4.2, ESI).

Agreement is seen between the values of $C_{\text {ion }}$ and $R_{\text {ion }}$ determined from the equivalent circuit fit and the values derived from the inputs to the Driftfusion model, helping to validate our interpretation of the system. The frequency dependence of $Z_{\text {rec }}$ displayed in a Nyquist plot gives rise to a low frequency semicircle in agreement with the observations of Pockett et al.10. The details are illustrated in Figs S4 and S5. In addition to yielding the ionic conductivity from $R_{\text {ion, }}$ fitting of our model to the impedance measurement enables quantification of the $J_{\mathrm{s} 1}, C_{\mathrm{g}}, C_{\mathrm{ion}}$ and $f_{\mathrm{c}}$ parameters. $C_{\mathrm{g}}, C_{\mathrm{ion}}$ and $f_{\mathrm{c}}$ are related to both the concentration of mobile ionic charge in the perovskite, and the concentration of dopants in the contacts as well as the dielectric constants of the materials (excluding any apparent contributions to the dielectric constant from surface polarisation by mobile ions). These control where electrostatic potential drops and, in combination with $R_{\text {ion, }}$, the magnitude, and timescale of hysteresis effects that a given cell will produce. The saturation current density, $J_{\mathrm{s} 1}$ (with $m_{1}$, determined from the steady state ideality factor, and $f_{\mathrm{c}}$ ) parameterises non-radiative recombination at the interface and is likely to be related to the density and depth of interfacial traps, a factor critical for assessing the relative performance of different interface combinations.

The expression we have derived for $Z_{\text {rec }}$ (equation 4) explains the majority of unusual features observed in the impedance spectroscopy measurements of hybrid perovskite solar cells. Similar arguments can be used to derive expressions for the impedance to recombination of holes at the perovskite/ETM interface which also yield capacitive behaviour (see Methods section S4 and the general case in Note S6, ESI). However, in some perovskite devices, inductor-like behaviour is seen in their impedance spectra ${ }^{11,19}$ and is also apparent in the slow evolution of current towards a new steady state in response to step changes of voltage or light ${ }^{4}$. The capacitor-like form of $Z_{\text {rec }}$ in equation 3 is unable to explain this inductive behaviour.

The description of the electronic impedance so far assumed that the rate of injection and collection is sufficiently fast (also shown by $V_{\mathrm{n}} \approx 0$ ) such that the electronic impedance is dominated by the recombination process (Fig. $4 \mathrm{~b}$ and c). If this were not the case, the electron injection $\left(U_{\mathrm{inj}}\right)$ and collection $\left(U_{\mathrm{col}}\right)$ currents at interface 2 follow an analogous dependence on the injection and collection voltages $V_{\text {inj }}$ and $V_{\text {col }}$ which are controlled by the electrostatic potential $V_{2}$ Table 1, see Notes S1 and S2, ESI, and Fig. 4e).

In the limiting case where charge injection dominates the impedance of the circuit, at low frequencies, the out of phase injection current is negatively amplified by the ionic current (hypothetical examples are shown in Fig. S4c and d, ESI). The trans-carrier amplification factor is $-R_{\mathrm{ion}} / 2\left[q J_{\mathrm{inj}}(V, \omega=0) / k_{\mathrm{B}} T\right]$ (c.f. equation 3) resulting in inductive behaviour (see Note $\mathrm{S} 1$, ESI). The effect opens the possibility to design thin film devices with huge tuneable effective 
inductances per unit volume $\left(>10^{4} \mathrm{H} \mathrm{cm}^{-3}\right)$ without relying on the elements coupling to a magnetic flux.

Given the influence of the ionic circuit on the electronic impedance described here, we note that more complex interactions of ionic charge with electronic charge or contact materials would also modulate interfacial electronic processes (see Note S2, Figs S1 and S6, ESI for effects of both interfaces). For example, the phase of $j_{\text {rec }}$ can lag $v$ if ionic charge penetrates, or undergoes a reversible chemical reaction, at a dominant recombination interface. Fits from an equivalent circuit allowing ion penetration into an interface to experimental data are shown in Fig. 5a. The ion penetration/reversible reaction is approximated by extending the ionic circuit branch into one of the contacts with an additional interfacial ionic transfer (or reaction) resistance $\left(R_{\text {int }}\right)$ and an ionic capacitance within the contact $\left(C_{\mathrm{ch}}\right)$. Under these circumstances our transistor interface model implies that the ionic gating of the electronic recombination process can result in both apparent capacitive and inductive behaviour.

a Experimental + Circuit Model

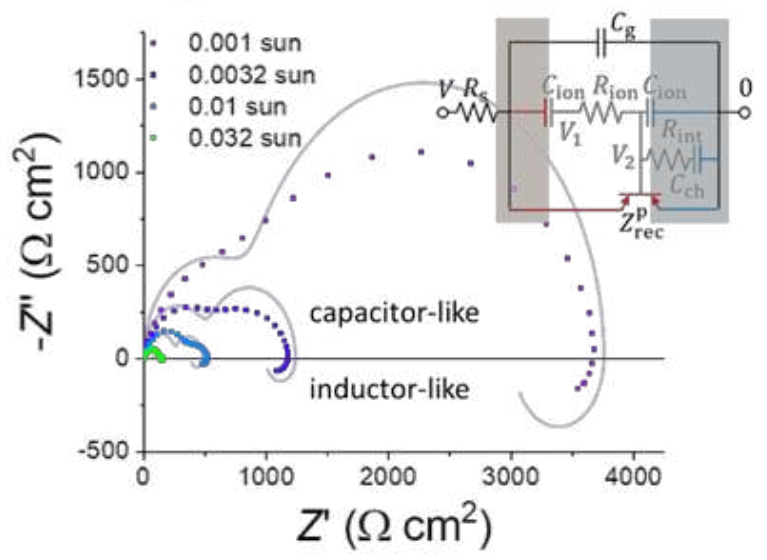

c Experimental

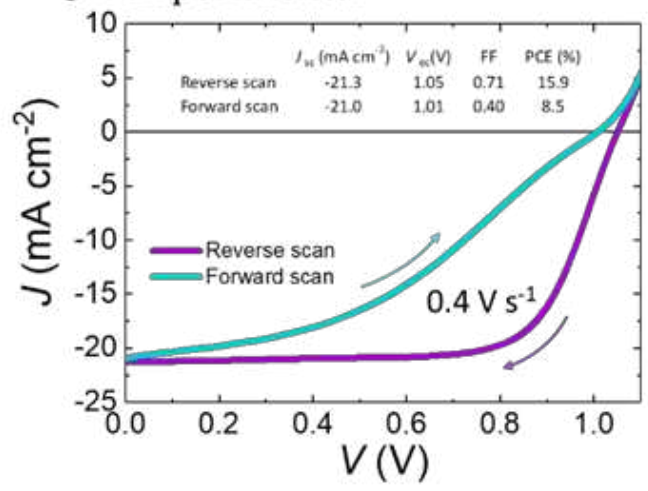

b Simulated + Circuit Model (Fig. 4g)

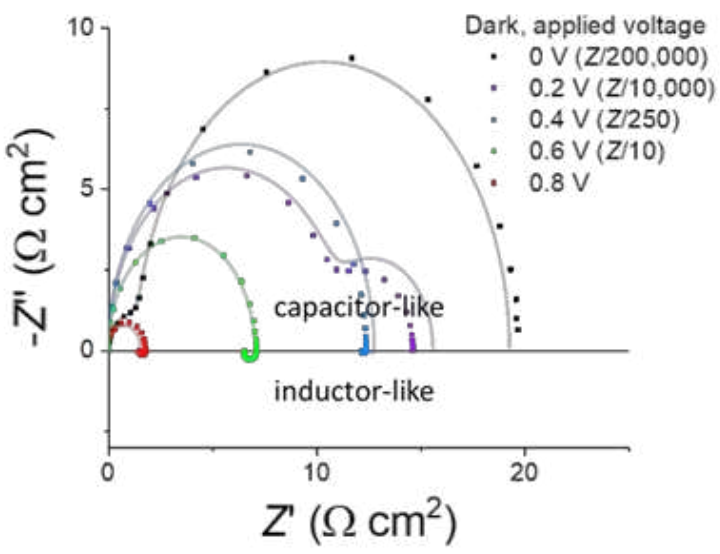

d Circuit Model

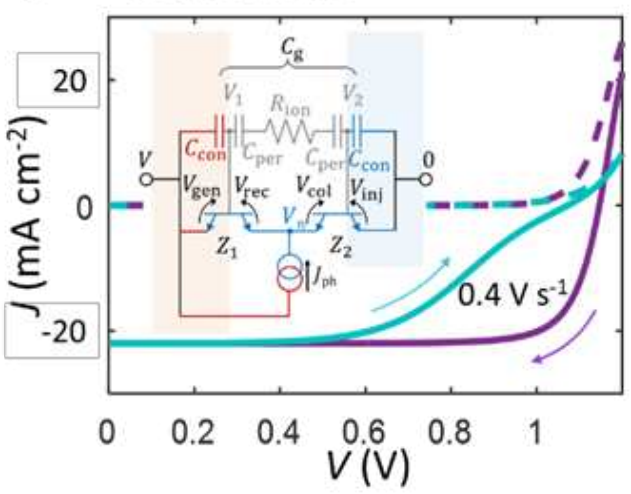

Fig. 5 Measurements, simulations, and models of different devices showing inductive behaviour and current-voltage behaviour. $\mathbf{a}, \mathbf{b}$, Nyquist plot of the real (Z') vs imaginary ( $\left.Z^{\prime \prime}\right)$ impedance components (filled squares) for (a) a spiro-OMeTAD/ $\mathrm{FA}_{0.85} \mathrm{MA}_{0.15} \mathrm{PbI}_{3} / \mathrm{SnO}_{2}$ solar cell (Methods 1.2, ESI) measured around the open circuit voltage, illuminated at different constant light intensities and (b) a drift-diffusion simulated (different) device with low majority carrier mobility in contacts and high 
interfacial recombination in the dark. The inset in a shows the equivalent circuit model used for the global fit to the data, note that the ionic circuit branch is a crude approximation allowing penetration and/or reversible reaction of ions at interface 2 . The equivalent circuit model used to fit the simulated data in $\mathbf{b}$ is shown in Fig. 4g. The solid lines are global fits to the data using 8 and 6 free parameters respectively (see Table S1, ESI) and the models with all data are shown in Fig. S7, ESI. (c) Measured current-voltage characteristics for the device characterised in Fig. 2a and b with forward and reverse voltage scans at $0.4 \mathrm{~V} \mathrm{~s}^{-1}$ under an AM1.5 solar spectrum. (d) Modelled light (solid lines where $J_{\mathrm{ph}}=22 \mathrm{~mA} \mathrm{~cm}^{-2}$ ) and dark (dashed lines) current-voltage characteristics using the inset equivalent circuit (similar to Fig. $4 \mathrm{~g}$ but with photocurrent generation included explicitly, and we have explicitly shown $C_{\text {ion }}=$ $\left(C_{\text {con }}^{-1}+C_{\text {per }}^{-1}\right)^{-1}$ with $f_{\mathrm{c}}=C_{\text {ion }} / C_{\text {con. }}$. The input parameters were determined from the fit of this model to the impedance data shown in Fig. 2a and b. The global fits, using 6 free parameters are shown in Fig. S1 (ESI), with the parameters given in Table S1 (ESI). Further circuit modelled $J-V$ curves for different scan rates are shown in Fig. S6 (ESI). These current sweeps are calculated using the approach described in Note S4.

We emphasise that the transistor element was used in the circuit model but not the simulations. The circuit model described encapsulates the key physical processes observed in simulations based on the standard current continuity equations, charge transfer processes (generation, recombination, collection and injection), and Poisson's equation with mobile ions having a higher concentration and lower conductivity than electronic charge under operation. The gating of interfacial electronic charge transfer (and thus electronic current through the device) by ionic redistribution (and consequent surface polarisation) explains very high low frequency apparent capacitances and inductances without accumulation of electronic charge at the interfaces. In contrast, the surface polarisation model introduced by Bisquert et al. ${ }^{17-19}$ requires that large concentrations of electronic charge accumulate at the interfaces to explain observed cell behaviour. If this were the case, significantly lower $V_{\text {OC }}$ values than typically observed in these devices might be expected.

Our model provides a basis to include additional factors that may influence device behaviour such as: the fraction of ionic screening potential dropping within the HTM and ETM contacts, asymmetric interfacial ionic capacitances, non-ideal recombination and injection (Methods 4.2, ESI), treatment of both electrons and holes (Fig. 4h), recombination in the perovskite bulk, and the effect of interface screening by electronic charge (see Fig. S8d-f, Notes S6 and S3, ESI). The latter factor is expected to be relevant in record efficiency solar cells and at large light or electrical bias conditions. In its simplest version, our ionically coupled transistor circuit model is already able to interpret the most important features of impedance spectra observed in the literature. Additionally, it also allows simple calculation of large perturbation measurements such as J-V sweeps at any scan speed (see Fig. $5 c$ and d, Fig. S6 and Note S4, ESI) and voltage step measurements (Note S5, ESI) as well as the d.c. (photo)current of the device. Such transient 
outputs of the circuit model could be used to parameterise measurements of device current or voltage response to voltage or light intensity steps which have been used previously to assess the influence of mobile ions. $4,26,32$

In cases where the impedance from each interface in the circuit model is comparable, an analytical solution is no longer accessible due to the need to numerically evaluate $V_{\mathrm{n}}$ (and/or $V_{\mathrm{p}}$ ). However, the procedure to determine the device behaviour is qualitatively similar and straightforward (see Notes S2 and S6, ESI); an example of a fit using numerical evaluation of $V_{\mathrm{n}}$ to a drift-diffusion simulated device with mixed capacitor and inductor-like behaviour is shown in Fig. 5b.

To conclude, our description of the interfaces of perovskite devices as ionically gated transistors provides an intuitive framework to interpret the complicated current-voltage behaviour of these devices as well as unlocking the potential of impedance spectroscopy as a means to identify the key bottlenecks of their performance. The interfacial transistor model also has a number of interesting broader implications. The trans-carrier amplification phenomenon described suggests a strategy to design devices displaying huge, tuneable, effective capacitances or inductances without the volume required for similar physical capacitances or inductances and with the option to be powered by light. Furthermore, the model will be generally applicable to other electrochemical redox processes supported by a high concentration of low mobility inert ions as well as to ionic motion signal sensing and amplification in biological systems requiring neural interfacing in a manner related to electrochemical transistors. ${ }^{40}$

\section{References}

1. J. Nelson, The Physics of Solar Cells, Imperial College Press, London, 2003.

2. M. M. Lee, J. Teuscher, T. Miyasaka, T. N. Murakami and H. J. Snaith, Science, 2012, 338, 643-647.

3. H.-S. Kim, C.-R. Lee, J.-H. Im, K.-B. Lee, T. Moehl, A. Marchioro, S.-J. Moon, R. HumphryBaker, J.-H. Yum, J. E. Moser, M. Grätzel and N.-G. Park, Scientific Reports, 2012, 2, 591.

4. C. Eames, J. M. Frost, P. R. F. Barnes, B. C. O'Regan, A. Walsh and M. S. Islam, Nat Commun, 2015, 6, 7497.

5. Z. Xiao, Y. Yuan, Y. Shao, Q. Wang, Q. Dong, C. Bi, P. Sharma, A. Gruverman and J. Huang, Nat Mater, 2015, 14, 193-198.

6. Y. Yuan and J. Huang, Accounts of Chemical Research, 2016, 49, 286-293.

7. G. Gregori, T.-Y. Yang, A. Senocrate, M. Grätzel and J. Maier, in Organic-Inorganic Halide Perovskite Photovoltaics: From Fundamentals to Device Architectures, eds. N.-G. Park, M. Grätzel and T. Miyasaka, Springer International Publishing, Cham, 2016, pp. 107-135.

8. A. Guerrero, G. Garcia-Belmonte, I. Mora-Sero, J. Bisquert, Y. S. Kang, T. J. Jacobsson, J.-P. Correa-Baena and A. Hagfeldt, The Journal of Physical Chemistry C, 2016, 120, 8023 8032.

9. T. Kazuya, Applied Physics Express, 2017, 10, 059101. 
10. A. Pockett, G. E. Eperon, N. Sakai, H. J. Snaith, L. M. Peter and P. J. Cameron, Phys. Chem. Chem. Phys., 2017, 19, 5959-5970.

11. O. Almora, K. T. Cho, S. Aghazada, I. Zimmermann, G. J. Matt, C. J. Brabec, M. K. Nazeeruddin and G. Garcia-Belmonte, Nano Energy, 2018, 48, 63-72.

12. C. Ludmila, U. Satoshi, V. V. J. Piyankarage, K. Shoji, T. Yasutake, N. Jotaro, K. Takaya and S. Hiroshi, Applied Physics Express, 2017, 10, 025701.

13. E. J. Juarez-Perez, R. S. Sanchez, L. Badia, G. Garcia-Belmonte, Y. S. Kang, I. Mora-Sero and J. Bisquert, The Journal of Physical Chemistry Letters, 2014, 5, 2390-2394.

14. A. Dualeh, T. Moehl, N. Tétreault, J. Teuscher, P. Gao, M. K. Nazeeruddin and M. Grätzel, Acs Nano, 2014, 8, 362-373.

15. M. N. F. Hoque, M. Yang, Z. Li, N. Islam, X. Pan, K. Zhu and Z. Fan, ACS Energy Letters, 2016, 1, 142-149.

16. O. Almora, I. Zarazua, E. Mas-Marza, I. Mora-Sero, J. Bisquert and G. Garcia-Belmonte, The Journal of Physical Chemistry Letters, 2015, 6, 1645-1652.

17. I. Zarazua, J. Bisquert and G. Garcia-Belmonte, The Journal of Physical Chemistry Letters, 2016, 7, 525-528.

18. I. Zarazua, G. Han, P. P. Boix, S. Mhaisalkar, F. Fabregat-Santiago, I. Mora-Seró, J. Bisquert and G. Garcia-Belmonte, The Journal of Physical Chemistry Letters, 2016, 7, 5105-5113.

19. E. Ghahremanirad, A. Bou, S. Olyaee and J. Bisquert, The Journal of Physical Chemistry Letters, 2017, 8, 1402-1406.

20. P. Schulz, E. Edri, S. Kirmayer, G. Hodes, D. Cahen and A. Kahn, Energy Environ. Sci., 2014, 7, 1377-1381.

21. W. Shockley, The Bell System Technical Journal, 1949, 28, 435-489.

22. D. Moia, I. Gelmetti, P. Calado, W. Fisher, M. Stringer, O. Game, Y. Hu, P. Docampo, D. Lidzey, E. Palomares, J. Nelson and P. R. F. Barnes, arXiv, 2018, 1805.06446

23. D. Prochowicz, P. Yadav, M. Saliba, M. Saski, S. M. Zakeeruddin, J. Lewiński and M. Grätzel, ACS Applied Materials \& Interfaces, 2017, 9, 28418-28425.

24. Y.-C. Zhao, W.-K. Zhou, X. Zhou, K.-H. Liu, D.-P. Yu and Q. Zhao, Light: Science \&Amp; Applications, 2017, 6, e16243.

25. G. Y. Kim, A. Senocrate, T.-Y. Yang, G. Gregori, M. Grätzel and J. Maier, Nat Mater, 2018, 17, 445-449.

26. P. Calado, A. M. Telford, D. Bryant, X. Li, J. Nelson, B. C. O’Regan and P. R. F. Barnes, Nat Commun, 2016, 7, 13831.

27. P. Calado, I. Gelmetti, M. Azzouzi, B. Hilton and P. R. F. Barnes, https://github.com/barnesgroupICL/Driftfusion, 2018.

28. G. Richardson, S. E. J. O'Kane, R. G. Niemann, T. A. Peltola, J. M. Foster, P. J. Cameron and A. B. Walker, Energy Environ. Sci., 2016, 9, 1476-1485.

29. S. van Reenen, M. Kemerink and H. J. Snaith, The Journal of Physical Chemistry Letters, $2015,6,3808-3814$.

30. W. Tress, N. Marinova, T. Moehl, S. M. Zakeeruddin, M. K. Nazeeruddin and M. Gratzel, Energy Environ. Sci., 2015, 8, 995-1004. 
31. Y. Wu, H. Shen, D. Walter, D. Jacobs, T. Duong, J. Peng, L. Jiang, Y.-B. Cheng and K. Weber, Adv. Funct. Mater., 2016, 26, 6807-6813.

32. D. Walter, A. Fell, Y. Wu, T. Duong, C. Barugkin, N. Wu, T. White and K. Weber, The Journal of Physical Chemistry C, 2018, 122, 11270-11281.

33. D. A. Jacobs, H. Shen, F. Pfeffer, J. Peng, T. P. White, F. J. Beck and K. R. Catchpole, Journal of Applied Physics, 2018, 124, 225702.

34. G. Xing, N. Mathews, S. Sun, S. S. Lim, Y. M. Lam, M. Grätzel, S. Mhaisalkar and T. C. Sum, Science, 2013, 342, 344-347.

35. S. D. Stranks, G. E. Eperon, G. Grancini, C. Menelaou, M. J. P. Alcocer, T. Leijtens, L. M. Herz, A. Petrozza and H. J. Snaith, Science, 2013, 342, 341-344.

36. D. W. deQuilettes, W. Zhang, V. M. Burlakov, D. J. Graham, T. Leijtens, A. Osherov, V. Bulović, H. J. Snaith, D. S. Ginger and S. D. Stranks, Nature Communications, 2016, 7, 11683.

37. L. Contreras-Bernal, M. Salado, A. Todinova, L. Calio, S. Ahmad, J. Idígoras and J. A. Anta, The Journal of Physical Chemistry C, 2017, 121, 9705-9713.

38. P. Calado, D. Burkitt, J. Yao, J. Troughton, T. M. Watson, M. J. Carnie, A. M. Telford, B. C. O' Regan, J. Nelson and P. R. F. Barnes, Phs. Rev. Appl., 2019, in press

39. O. Mangla and V. Gupta, Journal of Materials Science: Materials in Electronics, 2016, 27, 12527-12532.

40. J. Rivnay, S. Inal, A. Salleo, R. M. Owens, M. Berggren and G. G. Malliaras, Nature Reviews Materials, 2018, 3, 17086.

\section{Acknowledgements}

We thank the EPSRC for funding this work (EP/J002305/1, EP/M025020/1, EP/M014797/1, EP/L016702/1, EP/P02484X/1, EP/R020574/1, EP/R023581/1). I.G. and E.P. would like to thank the MINECO for the CTQ2016-80042-R project. E.P. also acknowledges AGAUR for the SGR project 2014 SGR 763 and ICREA for financial support.

\section{Author contributions}

I.G., D.M. and P.B. initiated the project led by P.B. D.M. measured devices fabricated and developed by M.S., O.G., H.H., D.L. and P.D.; I.G. performed the simulations on software developed with P.C.; D.M. and P.B. developed the transistor description and circuit models; W.F. and D.M. performed the equivalent circuit fitting using circuit models coded by P.B. All authors discussed the results and participated in preparation of the manuscript drafted by P.B, D.M. I.G. and P.C.

\section{Competing interests}

I.G., D.M., P.C. and P.B. have filed a patent application based on aspects of this work.

\section{Materials \& Correspondence}


Correspondence and requests for materials should be addressed to P.B.

piers.barnes@imperial.ac.uk, D.M. davide.moia11@imperial.ac.uk, and I.G.

igelmetti@iciq.es . The simulation code is available at

https://github.com/barnesgroupICL/Driftfusion 